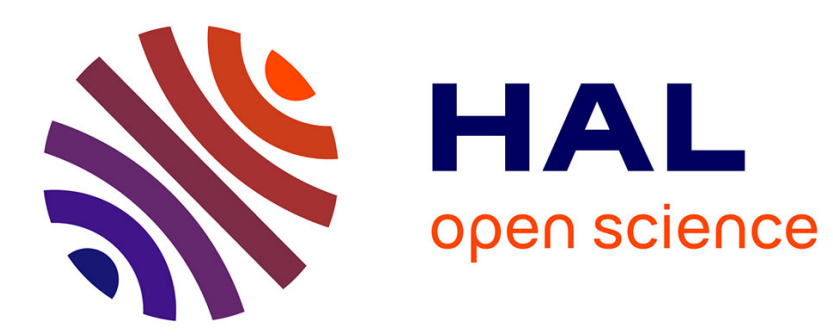

\title{
Multi-Lepton Events and Doubly-Charged Higgs Production at HERA
}

Corentin Vallée

\section{To cite this version:}

Corentin Vallée. Multi-Lepton Events and Doubly-Charged Higgs Production at HERA. 14th International Workshop on Deep Inelastic Scattering (DIS 2006), Apr 2006, Tsukuba, Japan. in2p3-00126047

\section{HAL Id: in2p3-00126047 https://hal.in2p3.fr/in2p3-00126047}

Submitted on 23 Jan 2007

HAL is a multi-disciplinary open access archive for the deposit and dissemination of scientific research documents, whether they are published or not. The documents may come from teaching and research institutions in France or abroad, or from public or private research centers.
L'archive ouverte pluridisciplinaire HAL, est destinée au dépôt et à la diffusion de documents scientifiques de niveau recherche, publiés ou non, émanant des établissements d'enseignement et de recherche français ou étrangers, des laboratoires publics ou privés. 


\title{
MULTI-LEPTON EVENTS AND DOUBLY-CHARGED HIGGS PRODUCTION AT HERA
}

\author{
CLAUDE VALLEE ${ }^{\dagger}$ \\ Centre de Physique des Particules de Marseille, CNRS-IN2P3, Université de la \\ Méditerranée, 163 Avenue de Luminy, F-13288 Marseille cedex 09, France
}

\begin{abstract}
Multi-lepton production at high transverse momentum is investigated by the $\mathrm{H} 1$ Collaboration in $\mathrm{e}^{+} \mathrm{p}$ and $\mathrm{e}^{-} \mathrm{p}$ collisions recorded at HERA until the end of 2005, corresponding to an integrated luminosity of $275 \mathrm{pb}^{-1}$. All event topologies involving electrons and muons are analysed. Di-lepton and tri-lepton event yields are found in general good agreement with the Standard Model predictions, dominated by $\gamma-\gamma$ interactions. In $\mathrm{e}^{+} \mathrm{p}$ collisions events with leptons of high transverse momenta are observed in a domain where the Standard Model prediction is low. Based on these topologies, a search for single production of doubly-charged Higgs bosons $\left(H^{ \pm \pm}\right)$ decaying into a high mass pair of same charge leptons is performed. No evidence for doubly-charged Higgs production is found and mass-dependent upper limits on the Yukawa couplings of the $H^{ \pm \pm}$to $e e, e \mu$ and $e \tau$ pairs are derived.
\end{abstract}

\section{Muti-lepton event production}

Within the Standard Model (SM) the production of multi-lepton events at high transverse momentum in ep collisions mainly proceeds via photon-photon interactions ${ }^{1}$. Precise cross-section measurements of both electron $(e)$ and muon $(\mu)$ pair production at high transverse momentum $\left(P_{T}\right)$ have already been performed by the $\mathrm{H} 1$ collaboration using the HERA I data ${ }^{2,3}$. At large dielectron masses, an excess of events was observed in both the di-electron and tri-electron samples ${ }^{2}$. The present analysis extends our previous measurements to the $e \mu$ and $e \mu \mu$ topologies and uses a higher integrated luminosity, combining new HERA II data taken in $e^{+} p$ collisions $\left(\mathbb{L}=52 \mathrm{pb}^{-1}\right)$ and in $e^{-} p$ collisions $(\mathbb{L}=$ $\left.105 \mathrm{pb}^{-1}\right)$ during the years 2003-2005 with the HERA I data sample from 1994$2000\left(\mathbb{L}=118 \mathrm{pb}^{-1}\right)$.

The multi-lepton selection requires at least two central $\left(20^{\circ}<\theta<150^{\circ}\right)$ lepton candidates ( $e$ or $\mu$ ) of which one must have $P_{T}>10 \mathrm{GeV}$ and the other $P_{T}$ $>5 \mathrm{GeV}$. Additional $e$ candidates are identified in the detector with an energy above $5 \mathrm{GeV}$ in the range $5^{\circ}<\theta<175^{\circ}$. Additional muons with $P_{T}>2 \mathrm{GeV}$ in the range $20^{\circ}<\theta<160^{\circ}$ are also looked for. The selected events are classified as $e e, \mu \mu, e \mu$, eee and $e \mu \mu$ as function of the number and flavours of the identified

${ }^{\dagger}$ On behalf of the H1 Collaboration. 
leptons. The observed event yields are given in Table 1. They are in good agreement with the SM expectations.

Table 1. Observed and predicted event yields for the multi-lepton samples. The SM predictions add contributions from the signal (Pair Production) and background (Neutral Current and Compton) processes. The errors on the predictions include model uncertainties and experimental systematic errors added in quadrature.

H1 Preliminary $275 \mathrm{pb}^{-1}(1994-2005)$

\begin{tabular}{|c|c|c|c|c|}
\hline Selection & Data & SM & Pair Production & NC-DIS + Compton \\
\hline $\mathrm{e}$ & 266 & $261 \pm 37$ & $217 \pm 23$ & $44 \pm 22$ \\
$\mu \mu$ & 113 & $112 \pm 21$ & $112 \pm 21$ & - \\
$\mathrm{e} \mu$ & 137 & $136 \pm 21$ & $83 \pm 6.5$ & $53 \pm 16$ \\
\hline $\mathrm{ee}$ & 52 & $52 \pm 6$ & $52 \pm 6$ & - \\
$\mathrm{e} \mu \mu$ & 63 & $67 \pm 10.5$ & $67 \pm 10.5$ & - \\
\hline
\end{tabular}

Figure 1 shows the distribution of the scalar sum $\left(\Sigma P_{T}\right)$ of $P_{T}$ of all identified leptons for the $e^{+} p, e^{-} p$ and combined data samples. For $\Sigma P_{T}>100$ $\mathrm{GeV} 4$ events are observed while $1.1 \pm 0.2$ are expected in overall. These four data events correspond to the three high mass ee events observed in the HERA I data $^{2}$ and to one new e $\mu \mu$ event observed in the HERA II data. All events with $\Sigma P_{T}>100 \mathrm{GeV}$ have been recorded in the $e^{+} p$ collisions.

\section{Search for doubly-charged Higgs}

Doubly-charged Higgs bosons $\left(H^{ \pm \pm}\right)$appear in various extensions of the SM in which the usual Higgs sector is extended by one or more triplet(s) with non-zero hypercharge ${ }^{4}$. The Higgs triplet(s) may couple to lepton fields via Yukawa couplings which are not constrained to be small since they are not involved in the mass generation. A non-vanishing coupling of a doubly-charged Higgs to an electron-lepton pair would allow its single production in ep collisions at HERA. With unpolarized incident beams the production is insensitive to the helicity structure of the Yukawa couplings. The present analysis ${ }^{5}$ investigates $H^{ \pm \pm}$ decays into $e e, e \mu$ and $e \tau$ pairs using the unpolarized HERA I data.

Searches in the $e e$ and $e \mu$ channels are based on published analyses ${ }^{2,3}$. The $e \tau$ channel is investigated on a subset of the HERA I data $\left(\mathbb{L}=88 \mathrm{pb}^{-1}\right)$ in the phase space $P_{T}^{\mathrm{e}, \tau}>10,5 \mathrm{GeV}$ and $20^{\circ}<\theta_{\mathrm{e}, \tau}<140^{\circ}$, considering all possible e, $\mu$ and hadronic decays of the $\tau$ lepton. After the final Higgs selection criteria no significant excess over the SM expectation is observed in the data. Figure 2 shows the upper limits derived on the $H^{ \pm \pm}$production cross sections times decay branching ratio, together with the corresponding limits on the Yukawa couplings $h_{e e}, h_{e \mu}$ and $h_{e \tau}$ obtained by assuming that one single coupling dominates. Bounds from the $\operatorname{LEP}^{6}$ and TEVATRON ${ }^{7}$ experiments are also shown. 

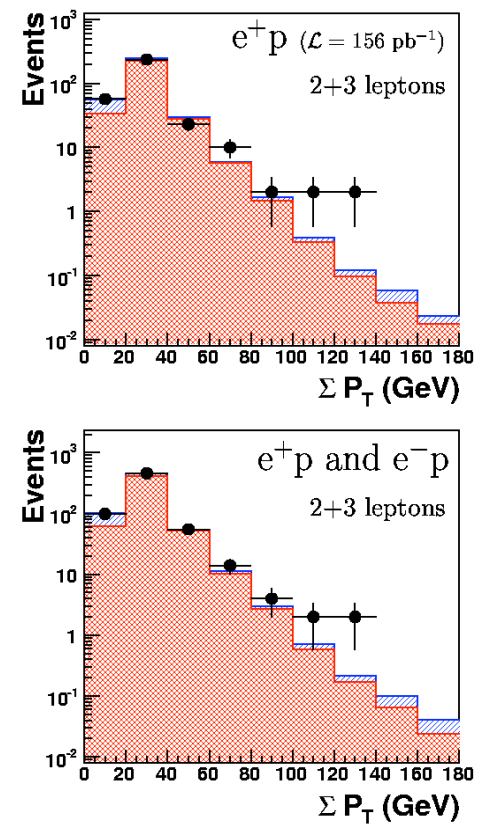

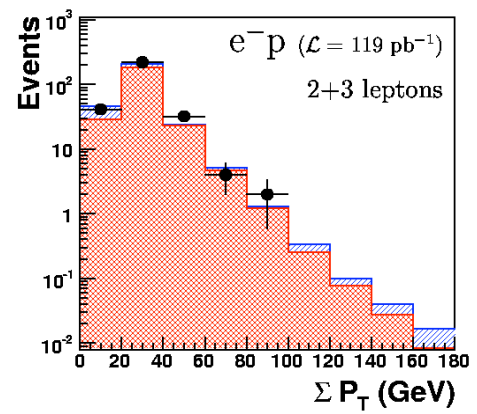

H1 Data (prelim.)
DIS+Compton

Pair Production

Figure 1. Distributions of the scalar sum $\Sigma P_{T}$ of the transverse momenta of identified leptons compared to expectations for $e^{+} p$ data (top left), $e^{-} p$ data (top right) and combined data (bottom).

In the ee channel $H^{ \pm \pm}$masses below $138 \mathrm{GeV}$ are excluded for a coupling $h_{e e}$ of the electromagnetic strength, $h_{e e}=0.3$. Amongst the six events at $M_{e e}>$ $100 \mathrm{GeV}$ observed in ${ }^{2}$ only one satisfies the $H^{ \pm \pm}$selection criteria, which makes the $H^{ \pm}$interpretation unlikely for these events. In the $e \mu$ (resp. $e \tau$ ) channel masses below $141 \mathrm{GeV}$ (resp. $112 \mathrm{GeV}$ ) are excluded for a Yukawa coupling of 0.3. In these channels H1 significantly extends the previous excluded domains.

\section{References}

1. J.A.M. Vermaseren, Nucl. Phys. B 229, (1983) 347;

N. Artega-Romero, C. Carimalo and P. Kessler, Zeit.f.Phys C 52, (1991) 289.

2. A. Aktas et al. [H1 Collaboration], Eur. Phys. J. C 31 (2003) 17 [hep-ex/0307015].

3. A. Aktas et al. [H1 Collaboration], Phys. Lett. B 583 (2004) 28 [hep-ex/0311015].

4. G. B. Gelmini and M. Roncadelli, Phys. Lett. B 99 (1981) 411.

R. N. Mohapatra and G. Senjanovic, Phys. Rev. Lett. 44 (1980) 912.

B. Dutta and R. N. Mohapatra, Phys. Rev. D 59 (1999) 015018

[hep-ex/9804277] 

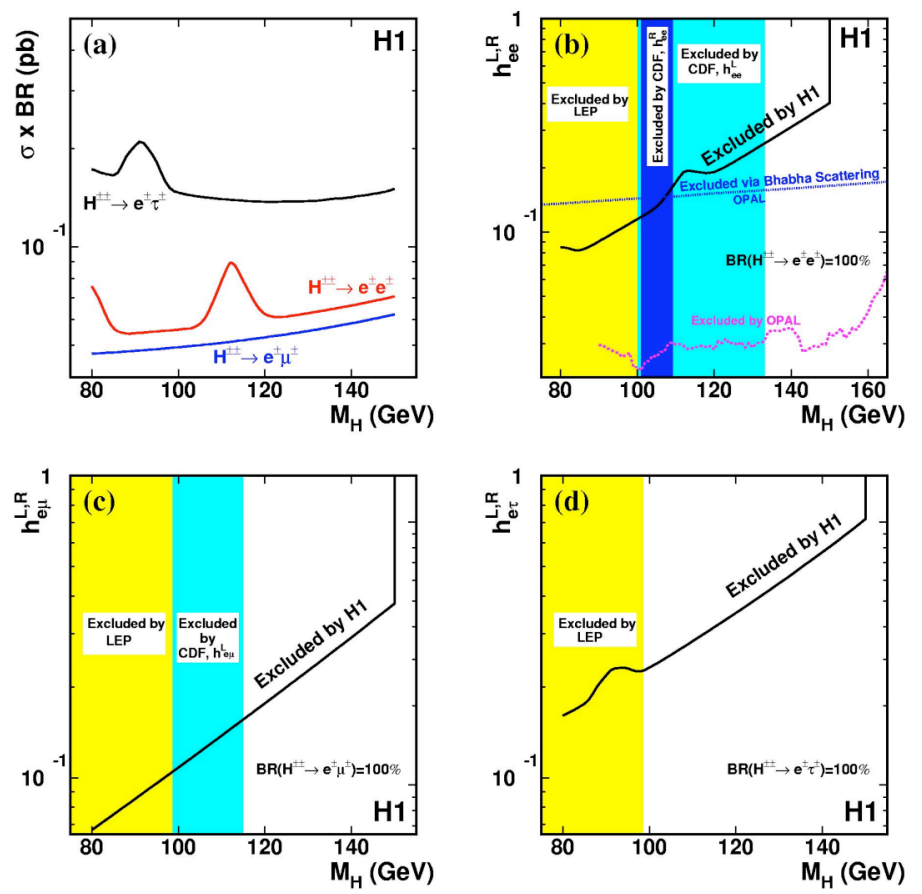

Figure 2. Upper limits at the $95 \%$ confidence level on the $H^{ \pm \pm}$production cross section times branching ratio (a), and on the couplings $h_{e l}$ assuming the $H^{ \pm \pm}$couples only to $e e$ (b), $e \mu$ (c) or $e \tau(\mathrm{d})$, as a function of the $H^{ \pm}$mass $\mathrm{M}_{\mathrm{H}}$.

5. A. Aktas et al. [H1 Collaboration], DESY 06-038 and hep-ex/0604027, submitted to Phys. Lett. B.

6. J. Abdallah et al. [DELPHI Collaboration], Phys. Lett. B 552 (2003) 127 [hep-ex/0303026];

P. Achard et al. [L3 Collaboration], Phys. Lett. B 576 (2003) 18

[hep-ex/0309076];

G. Abbiendi et al. [OPAL Collaboration], Phys. Lett. B 526 (2002) 221

[hep-ex/0111059].

G. Abbiendi et al. [OPAL Collaboration], Phys. Lett. B 577 (2003) 93 [hep-ex/0308052].

7. D. Acosta et al. [CDF Collaboration], Phys. Rev. Lett. 93 (2004) 221802 [hep-ex/0406073]. 